\title{
Prehospital Protocols Reducing Long Spinal Board Use Are Not Associated with a Change in Incidence of Spinal Cord Injury
}

\section{Authors:}

1. Franco Castro-Marin MD $\dagger$

- fcmarin@honorhealth.com

2. Joshua B Gaither MD*

- jgaither@aemrc.arizona.edu

3. Amber D Rice MD*

- $\quad$ arice@aemrc.arizona.edu

4. Robyn Nicole Blust MPH**

- robyn.blust@azdhs.gov

5. Vatsal Chikani MPH**

- vatsal.chikani@azdhs.gov

6. Anne Vossbrink**

- anne.vossbrink@azdhs.gov

7. Bentley J Bobrow MD****

- bentley.bobrow@azdhs.gov

Corresponding Author:

Franco Castro-Marin MD

HonorHealth | Osborn Emergency Department

P.O. Box 2808

Scottsdale, AZ 85252

Phone: 480-882-4809

Fax: 480-882-4389

Email: fcmarin@honorhealth.com

$\uparrow$ HonorHealth | Osborn Emergency Department

*University of Arizona College of Medicine, Arizona Emergency Medicine Research Center, Department of Emergency Medicine

**Arizona Department of Health and Human Services, Phoenix, Arizona

Key Words: Spinal Immobilization; Spinal Cord Injury; Long Spinal Board; Spinal Motion Restriction; Emergency Medical Services 


\begin{abstract}
Introduction: Many EMS agencies have de-emphasized or eliminated the use of long spinal boards (LSB) for patients with possible spinal injury. We sought to determine if implementation of spinal motion restriction (SMR) protocols, which reduce LSB use, was associated with an increase in spinal cord injury (SCI).
\end{abstract}

Methods: This retrospective observational study includes EMS encounters from 1/1/2013 to 12/31/2015 submitted by SMR-adopting ground-based agencies to a state EMS database with hospital discharge data. Encounters were excluded if SMR implementation date was unknown, occurred during a 3-month run-in period, or were duplicates. Study samples include patients with traumatic injury (TI), possible spinal trauma (P-ST), and verified spinal trauma (V-ST) using hospital discharge ICD-9/10 diagnosis codes. The incidence of SCI before and after implementation of SMR was compared using Chi-squared and logistic regression.

Results: From 1,005,978 linked encounters, 104,315 unique encounters with traumatic injury and known SMR implementation date were identified with 51,199 cases of P-ST and 5,178 V-ST cases. The incidence of SCI in the pre-SMR and post-SMR interval for each group was: TI, $0.20 \%$ vs. $0.22 \%(\mathrm{p}=0.390)$; P-ST, $0.40 \%$ vs. $0.45 \%(\mathrm{p}=0.436)$; and V-ST, $4.04 \%$ vs. $4.37 \%(\mathrm{p}=$ 0.561). Age and injury severity adjusted odds ratio of SCI in the highest risk cohort of patients with V-ST was 1.097 after SMR implementation (95\% CI 0.818-1.472).

Conclusion: In this limited study, no change in the incidence of SCI was identified following implementation of SMR protocols. Prospective evaluation of this question is necessary to evaluate the safety of SMR protocols. 


\section{Introduction}

Traditional spinal immobilization (SI), defined as the combined use of a long spinal board (LSB) and cervical collar (c-collar), has been a mainstay of prehospital trauma care since the 1970s.[1] Both the safety and efficacy of traditional SI have come into question in recent years and as a result some emergency medical services (EMS) agencies have deemphasized the use of LSBs and c-collars.[2-5] These changes have been implemented based on new evidence that highlights the low incidence of spinal cord injury (SCI), potential harm from the application of these devices,[6-12] and lack of evidence demonstrating any positive effect on mortality, spinal stability, or neurologic injury.[5, 13] Several studies have highlighted specific associations between the use of SI devices and predictors of negative outcomes following trauma, including increased intracranial pressure with c-collar use after traumatic brain injury,[14] as well as increased time spent on scene by EMS personnel, decreased intubation success, and decreased respiratory capacity.[6-10, 15-18] Despite the goal of reducing the negative secondary effects of SI, the safety of a more selective approach to the use of c-collars and LSBs in the process of packaging, extrication, and transport of trauma patients, referred to as spinal motion restriction (SMR), remains unknown.

There are over 12 million emergency department visits for falls and motor vehicle accidents and only 10-20,000 new cases of SCI diagnosed annually in the United States,[19-21] creating a very low incidence of SCI in patients with trauma.[22] The lowest rates of unstable spinal injury are in patients with penetrating trauma, nearing $0.01 \%,[10,12]$ with slightly higher rates in a more general trauma cohort.[23-25] The highest rates of SCI, in multi-system trauma patients with high injury severity scores, approach 7.5\%.[17] This low incidence of SCI has formed the 
underpinning of SMR protocols. These protocols, applied to a wide range of trauma patients (both low- and high-risk), seek to minimize harm from SI while maintaining the possible, albeit empirically unproven benefit to high-risk patients.

Given the lack of evidence to support traditional SI as well as its potential complications, [2, 413, 15, 18, 26-35] many EMS systems have implemented SMR protocols. These protocols emphasize the use of the least restrictive immobilization techniques to be applied only to those patients with significant risk factors or abnormal findings on examination. The implementation of these protocols has resulted in a significant decrease in the number of patients transported using traditional SI procedures and equipment, particularly LSBs.[36-38] The objective of this study was to determine if any change in the incidence of SCI could be identified after the implementation of SMR protocols in multiple EMS systems across a single state.

\section{Materials and Methods}

\section{Data Sources and Study Groups:}

This was a statewide, retrospective, observational, multi-agency, prehospital study including encounters from January 1, 2013 to December 31, 2015. Linked prehospital and hospital data were obtained from the Arizona Prehospital Information and Emergency Medical Services Registry System (AZ-PIERS) with retrospective deterministic linkage of encounters to the Hospital Discharge Database (HDD) using a previously described method.[39] These data were made available to the researchers as a de-identified data set, compiled as part of a quality improvement project performed earlier by the Arizona Department of Health Services. No patient identifiers were available to the research team for this study. 
The Arizona Department of Health Services maintains both data sources. The AZ-PIERS, which is managed by the Department's Bureau of Emergency Medical Services and Trauma System, is a voluntary patient registry that allows EMS agencies to collect and transmit electronic Patient Care Records (ePCR) to the State. The database includes both required and optional reporting elements and data are validated to meet National EMS Information System (NEMSIS) standards. The AZ-PIERS captures agency information, patient demographics, response times, incident location, and treatment provided. The HDD collects inpatient and emergency department visits from all Arizona-licensed hospitals. The HDD does not capture information from federal healthcare facilities such as the Veteran's Administration, Department of Defense, or tribal hospitals.

Prehospital encounters available from the AZ-PIERS system based on the defined study period were linked to the HDD using a stepwise deterministic linkage algorithm with direct identifiers (first name, last name, date of birth, social security number, gender, date of incident/hospital admission, hospital name). Exclusion criteria were applied to the linked datasets to remove air ambulance transports, inter-facility transfers, and encounters from agencies with unknown SMR implementation date. When two or more EMS agencies were involved in a patient's care, the first responding agency report was included and any other duplicate records were removed.

All EMS agencies submitting data to AZ-PIERS during the study period were contacted via email or phone in order to determine whether or not they had implemented a SMR protocol. Those using SMR were then asked to provide the date of implementation. Of the 85 agencies 
included in this study, $16(18.8 \%)$ were unable to provide protocol information and $1(1.2 \%)$ additional agency was unable to verify SMR implementation date. These 17 agencies were excluded. Each encounter was designated as pre- versus post-SMR based on each agency's date of implementation. Encounters were excluded if they occurred during a three-month run-in period including the month of SMR protocol implementation, the month prior, and the month following. Agencies who did not implement SMR during the study period were included in the pre-SMR group only.

Three study groups were identified from the complete linked data set. Patients with a principal diagnosis of traumatic injury (ICD-9 code 800-959 or ICD-10 code S00-T34 or T79) were identified as the full study cohort, Traumatic Injury (TI). The Center for Disease Control and Prevention's ICD-9-CM Barell Matrix[40] and the Proposed Framework for ICD-10-CM Diagnosis Codes[41] were used to identify cases of traumatic injury in which there was Possible Spinal Trauma (P-ST), defined as cases with a diagnosis of trauma to the head, face or neck, spinal cord or vertebral column. Finally, cases with Verified Spinal Trauma (V-ST) were identified as those with a diagnosis of spinal cord injury, vertebral fracture or vertebral dislocation.

The primary outcome for this study was SCI diagnosed at discharge by an ICD-9 or ICD-10 hospital discharge code. Demographic characteristics of pre- and post-SMR cohorts were compared using descriptive statistics (Table 1). Relative risk and Chi-Square analyses were performed to compare the incidence of SCI before and after SMR implementation in the full study group as well as the two subgroups. Multivariate logistic regression was performed to 
adjust for age, race, gender, and injury severity score (ISS). Unadjusted as well as adjusted odds ratios (OR) for all cohorts were reported with 95\% CI. All analyses were performed using SAS software, version 9.4 (SAS Institute, Cary, NC). Statistical significance was assessed using a two-sided p-value of 0.05 .

The authors assumed that there would be some variation in the exact language contained in SMR protocols across the state. In order to assess any possible impact on study results, a sensitivity analysis was performed using data from only those agencies whose protocols could be reviewed by the authors (FCM, JG) to ensure they contained certain critical elements. An example of a typical SMR protocol is shown in Figure 1.

\section{Human Subjects Committee Review:}

This study was reviewed by the Arizona Department of Health Services Human Subjects Review Board and deemed exempt from IRB review.

\section{Results}

As illustrated in Figure 2, the AZ-PIERS contained EMS dataset was comprised of 1,123,178 encounters that occurred during the study period and a total of 1,005,978 (89.6\%) of those were successfully linked to a hospital encounter within the HDD. After excluding air ambulance transports $(n=30,996)$, no EMS agency identified on EMS encounter $(n=3,747)$, inter-facility transfers $(\mathrm{n}=238,085)$, agencies with unknown SMR status $(\mathrm{n}=41,098)$ and duplicate records $(\mathrm{n}=99,437)$ there were 592,615 records eligible for study inclusion. Of those, 110,395 had a primary diagnosis of injury/trauma at the time of hospital discharge. After excluding encounters 
occurring during the three-month run-in period there were 104,315 patients from 68 EMS agencies included in the full TI group with 39,919 (38.3\%) pre-SMR cases and 64,396 (61.7\%) post-SMR cases. From the identified group with TI there were 51,199 patients transported to a hospital by EMS with a CDC Barrell matrix diagnosis of trauma involving the head or spine forming the P-ST group and 5,178 patients were transported with an ICD-9/10 code of spinal fracture, spinal cord injury or spinal dislocation forming the V-ST group.

Patient demographics and hospital outcome measures are reported in Table 1. There were small but statistically significant differences in many of the available patient demographics. The postSMR cohort was in general slightly older, more likely to be white, have an ISS greater than 15 , and a mechanism of injury documented as fall. Of note, these differences were found in the TI and P-ST cohorts but not identified in the V-ST cohort. In the V-ST cohort, there were no statistically significant differences between the pre-SMR and post-SMR demographics, other than race. In this cohort, more patients were discharged to home in the pre-SMR group than in the post-SMR group $(53.2 \%$ vs. $49.6 \%, \mathrm{p}<0.0001)$. The differences noted were small and likely reached significance related to the large sample size.

As illustrated in Table 2, there was no significant change observed in the incidence of SCI following SMR protocol implementation. Within the full study cohort (TI) the rate of SCI was $0.20 \%$ before SMR implementation and $0.22 \%$ after implementation $(\mathrm{p}=0.390)$. Within the P-ST group the incidence of SCI was $0.40 \%$ prior to SMR and $0.45 \%$ after implementation of SMR $(\mathrm{p}=0.436)$. Within the group of patients with V-ST the incidence of SCI was $4.04 \%$ prior to SMR implementation and $4.37 \%$ after SMR implementation $(\mathrm{p}=0.561)$. The RR of SCI after 
implementation of the SMR protocol in this very high-risk subgroup (V-ST) was 1.080 (95\% CI; 0.827-1.420).

Results of the multivariate logistic regression analysis are shown in Table 3. In this analysis after adjusting for differences in severity of injury (ISS), age, gender, and race, no statistically significant differences in unadjusted or adjusted odds of SCI was found in any group. A sensitivity analysis (data not shown) was performed using cases from only those agencies using verified SMR protocols and the findings are consistent with those from the primary analysis, supporting the assumption that minor variability in protocol language or design was unlikely to have a significant effect on incidence of SCI after protocol implementation.

\section{Discussion}

The purpose of this study is to assess the noninferiority of prehospital protocols aimed at limiting LSB use as well as to provide a framework for future studies evaluating the safety of prehospital protocol changes for spinal immobilization in trauma patients. We found that after implementation of SMR protocols across multiple EMS agencies in Arizona, there was not a statistically significant increase in the incidence of SCI. Our findings were consistent among trauma patients in general as well as within the highest risk cohort in our study, patients with confirmed SCI. Separately published data demonstrate that implementation of this protocol reduced LSB use even among patients with clear symptoms of SCI in the prehospital setting.[42] These data suggest that EMS agencies implementing SMR protocols to selectively immobilize traumatically injured patients are not putting their patients at increased risk of SCI compared with patients who receive traditional SI. We did not detect a statically significant increase in SCI 
after SMR implementation in the age and severity adjusted analysis, indicating that differences in overall injury severity or age of the patients between the pre- and post-SMR cohorts did not affect the noninferiority of SMR as it relates to incidence of SCI.

The primary outcome for this study was the rate of SCI following implementation of an SMR protocol. Patient outcome data were available and a small increase in the rate of discharge to skilled nursing or long-term care facilities as well increase in mortality after implementation of SMR were observed. These data must be interpreted with thoughtful consideration as increasing overall injury severity or changing hospital discharge practices over the study period may have influenced these results. Given that implementation of an SMR protocol would not be expected to affect overall in-hospital mortality or hospital disposition, this study was not designed to evaluate these outcomes.

The overall incidence SCI was low in this study, ranging from $0.20 \%$ to $4.37 \%$. This finding is consistent with other reports.[17, 19-21, 25, 43, 44] As expected, patients in the V-ST subgroup had the highest incidence of SCI, with $4.04 \%$ and $4.37 \%$ of patients diagnosed in the pre- and post-SMR cohorts respectively. Within a sample where the incidence of an injury is low, it is difficult to perform a study large enough to determine if a significant change in the incidence of injury occurred. Given that this study describes the non-inferiority of an SMR protocol when compared to traditional SI, we estimate that this study would have detected a $1 \%$ change in the incidence of SCI with implementation of SMR considering the observed incidence and the study sample evaluated. Although this study did not detect a significant change in the incidence of SCI, it cannot eliminate the possibility that a larger study might identify a clinically and 
statistically significant increase in the incidence of SCI following implementation of a SMR protocol.

A recently published, joint position statement published by the ACS-COT, the National Association of EMS Physicians, and the American College of Emergency Physicians, emphasized that spinal injuries can occur in a noncontiguous manner, and recommend that the entirety of the spine should be stabilized whenever SMR is employed. Specifically, they state that SMR cannot be properly performed with a patient in a sitting position.[45] It should be noted that the SMR protocols included in this study did allow for patients to be transported in Fowler's or semi-Fowler's positions with only a cervical collar applied, with some consideration given to airway and respiratory status, patient comfort, and an overall achievement of reduced spinal motion.

There were several limitations to our study. First, these data did not contain information regarding how closely EMS personnel adhered to their agency's SMR protocol. Other data obtained from the same study sample suggest there was a significant decrease in the rate of LSB use after SMR protocol implementation within the highest risk subgroup of patients (those with SCI).[42] Second, this study relied on ICD-9/10 codes to determine patient outcomes and independent verification of acute SCI could not be performed. It is possible that a small number of cases had pre-existing spinal cord disease and an ICD-9/10 diagnosis code was entered into the patient record to indicate a previous injury. Third, inclusion in this study relied on EMS agency participation in a statewide, voluntary EMS patient registry, which could lead to inclusion of higher performing or larger EMS and better than expected patient outcomes. Fourth, 
despite a high case linkage rate (89.6\%), it is possible that we failed to identify some patients with SCI given the deterministic linkage methodology used. Finally, although a single set of SMR training materials was produced and distributed broadly, how each individual EMS agency delivered or modified that content could not be easily tracked. For this reason, the authors individually reviewed a subset of agency SMR protocols to ensure they contained certain common elements. It was not possible to review the protocols for every agency that participated in the study. We performed a sensitivity analysis using cases from only those agencies with verified SMR protocols to see if any variations might impact our study outcomes. The sensitivity analysis results were consistent with those of the primary outcome.

\section{Conclusion}

In conclusion, this study did not identify any significant increase in the incidence of SCI following implementation of an SMR protocol by multiple EMS agencies in a single state. This study adds to a growing body of evidence supporting the use of SMR techniques aimed at reducing the use of LSBs, but should not be used as the sole foundation to support the conclusion that SMR is safe. Further evaluation of SMR protocol safety is necessary before concluding that total elimination of the LSB is a safe practice. Future studies should focus on large-scale prospective evaluations of SMR protocols and quantify any associated risk or benefit. 


\section{References}

1. American Academy of Orthopaedic Surgeons. Committee on Injuries (1971). Emergency care and transportation of the sick and injured. The Academy, Chicago, Ill.

2. Kornhall DK, Jorgensen JJ, Brommeland T, Hyldmo PK, Asbjornsen H, Dolven T, et al. The Norwegian guidelines for the prehospital management of adult trauma patients with potential spinal injury. Scand J Trauma Resusc Emerg Med. 2017;25(1):2.

3. Theodore N, Hadley MN, Aarabi B, Dhall SS, Gelb DE, Hurlbert RJ, et al. Prehospital cervical spinal immobilization after trauma. Neurosurgery. 2013;72 Suppl 2:22-34.

4. Hood N, Considine J. Spinal immobilisaton in pre-hospital and emergency care: A systematic review of the literature. Australas Emerg Nurs J. 2015;18(3):118-37.

5. Kwan I, Bunn F, Roberts I. Spinal immobilisation for trauma patients. Cochrane Database Syst Rev. 2001(2):Cd002803.

6. Schafermeyer RW, Ribbeck BM, Gaskins J, Thomason S, Harlan M, Attkisson A. Respiratory effects of spinal immobilization in children. Ann Emerg Med. 1991;20(9):1017-9. 7. Raphael JH, Chotai R. Effects of the cervical collar on cerebrospinal fluid pressure. Anaesthesia. 1994;49(5):437-9.

8. Podolsky SM, Hoffman JR, Pietrafesa CA. Neurologic complications following immobilization of cervical spine fracture in a patient with ankylosing spondylitis. Ann Emerg Med. 1983;12(9):578-80.

9. Linares HA, Mawson AR, Suarez E, Biundo JJ. Association between pressure sores and immobilization in the immediate post-injury period. Orthopedics. 1987;10(4):571-3.

10. Cordell WH, Hollingsworth JC, Olinger ML, Stroman SJ, Nelson DR. Pain and tissueinterface pressures during spine-board immobilization. Ann Emerg Med. 1995;26(1):31-6. 
11. Chan D, Goldberg RM, Mason J, Chan L. Backboard versus mattress splint immobilization: a comparison of symptoms generated. J Emerg Med. 1996;14(3):293-8.

12. Bauer D, Kowalski R. Effect of spinal immobilization devices on pulmonary function in the healthy, nonsmoking man. Ann Emerg Med. 1988;17(9):915-8.

13. Kwan I, Bunn F. Effects of prehospital spinal immobilization: a systematic review of randomized trials on healthy subjects. Prehosp Disaster Med. 2005;20(1):47-53.

14. Kolb JC, Summers RL, Galli RL. Cervical collar-induced changes in intracranial pressure. Am J Emerg Med. 1999;17(2):135-7.

15. Chan D, Goldberg R, Tascone A, Harmon S, Chan L. The effect of spinal immobilization on healthy volunteers. Ann Emerg Med. 1994;23(1):48-51.

16. Kaups KL, Davis JW. Patients with gunshot wounds to the head do not require cervical spine immobilization and evaluation. J Trauma. 1998;44(5):865-7.

17. Stephan K, Huber S, Haberle S, Kanz KG, Buhren V, van Griensven M, et al. Spinal cord injury--incidence, prognosis, and outcome: an analysis of the TraumaRegister DGU. Spine J. 2015;15(9):1994-2001.

18. Totten VY, Sugarman DB. Respiratory effects of spinal immobilization. Prehosp Emerg Care. 1999;3(4):347-52.

19. DeVivo MJ. Causes and costs of spinal cord injury in the United States. Spinal Cord. 1997;35(12):809-13.

20. Jain NB, Ayers GD, Peterson EN, Harris MB, Morse L, O'Connor KC, et al. Traumatic spinal cord injury in the United States, 1993-2012. JAMA. 2015;313(22):2236-43. 
21. The epidemiology of spinal cord injury. In: Stover SL, DeLisa JA, Whiteneck GG (eds) Spinal Cord Injury: Clinical Outcomes from the Model Systems. Aspen Publishers: Gaithersburg, Maryland, 1995, pp $21 \pm 55$.

22. Niska R, Bhuiya F, Xu J. National Hospital Ambulatory Medical Care Survey: 2007 emergency department summary. Natn Health Stat Report. 2010(26):1-31.

23. Domeier RM, Swor RA, Evans RW, Hancock JB, Fales W, Krohmer J, et al. Multicenter prospective validation of prehospital clinical spinal clearance criteria. J Trauma. 2002;53(4):74450.

24. Hoffman JR, Mower WR, Wolfson AB, Todd KH, Zucker MI. Validity of a set of clinical criteria to rule out injury to the cervical spine in patients with blunt trauma. National Emergency X-Radiography Utilization Study Group. N Engl J Med. 2000;343(2):94-9.

25. Pirouzmand F. Epidemiological trends of spine and spinal cord injuries in the largest Canadian adult trauma center from 1986 to 2006. J Neurosurg Spine. 2010;12(2):131-40.

26. Ben-Galim P, Dreiangel N, Mattox KL, Reitman CA, Kalantar SB, Hipp JA. Extrication collars can result in abnormal separation between vertebrae in the presence of a dissociative injury. J Trauma. 2010;69(2):447-50.

27. Dunham CM, Brocker BP, Collier BD, Gemmel DJ. Risks associated with magnetic resonance imaging and cervical collar in comatose, blunt trauma patients with negative comprehensive cervical spine computed tomography and no apparent spinal deficit. Crit Care. 2008;12(4):R89.

28. Hamilton RS, Pons PT. The efficacy and comfort of full-body vacuum splints for cervical-spine immobilization. J Emerg Med. 1996;14(5):553-9. 
29. Johnson DR, Hauswald M, Stockhoff C. Comparison of a vacuum splint device to a rigid backboard for spinal immobilization. Am J Emerg Med. 1996;14(4):369-72.

30. Lerner EB, Billittier AJt, Moscati RM. The effects of neutral positioning with and without padding on spinal immobilization of healthy subjects. Prehosp Emerg Care. 1998;2(2):112-6.

31. Mawson AR, Biundo JJ, Jr., Neville P, Linares HA, Winchester Y, Lopez A. Risk factors for early occurring pressure ulcers following spinal cord injury. Am J Phys Med Rehabil. 1988;67(3):123-7.

32. Papadopoulos MC, Chakraborty A, Waldron G, Bell BA. Lesson of the week: exacerbating cervical spine injury by applying a hard collar. BMJ. 1999;319(7203):171-2.

33. Purvis TA, Carlin B, Driscoll P. The definite risks and questionable benefits of liberal pre-hospital spinal immobilisation. Am J Emerg Med. 2017;35(6):860-6.

34. Shafer JS, Naunheim RS. Cervical spine motion during extrication: a pilot study. West J Emerg Med. 2009;10(2):74-8.

35. Swartz EE, Nowak J, Shirley C, Decoster LC. A comparison of head movement during back boarding by motorized spine-board and log-roll techniques. J Athl Train. 2005;40(3):162-8. 36. Vaillancourt C, Stiell IG, Beaudoin T, Maloney J, Anton AR, Bradford P, et al. The outof-hospital validation of the Canadian C-Spine Rule by paramedics. Ann Emerg Med. 2009;54(5):663-71.

37. Morrissey JF, Kusel ER, Sporer KA. Spinal motion restriction: an educational and implementation program to redefine prehospital spinal assessment and care.Prehosp Emerg Care. 2014;18(3):429-32. 
38. Muhr MD, Seabrook DL, Wittwer LK. Paramedic use of a spinal injury clearance algorithm reduces spinal immobilization in the out-of-hospital setting. Prehosp Emerg Care. 1999;3(1):1-6.

39. Chikani V, Blust R, Vossbrink A, Wightman P, Bissell S, Graw J, et al. Improving the Continuum of Care by Bridging the Gap between Prehospital and Hospital Discharge Data through Stepwise Deterministic Linkage. Prehosp Emerg Care. 2019:1-7.

40. Bergen G CL, Warner M, Fingerhut LA. Injury in the United States: 2007 Chartbook. In: Statistics NCfH, editor. Hyattsville, MD2008.

41. Hedegaard H, Johnson RL, Warner M, Chen LH, Annest JL. Proposed Framework for Presenting Injury Data Using the International Classification of Diseases, Tenth Revision, Clinical Modification (ICD-10-CM) Diagnosis Codes. Natl Health Stat Report. 2016(89):1-20.

42. Gaither JB, Fletcher P, Rice A, Castro-Marin F, Blust R, Chikani V, Vossbrink A, Navaro T, Bobrow B. Does an Emergency Medical Services Protocol Decrease Spinal Board Use in Patients with Spinal Cord Injury? Acad Emerg Med. 2018;25(S1):1.

43. DeVivo MJ. Epidemiology of traumatic spinal cord injury: trends and future implications. Spinal Cord. 2012;50:365.

44. Kumar R, Lim J, Mekary RA, Rattani A, Dewan MC, Sharif SY, et al. Traumatic Spinal Injury: Global Epidemiology and Worldwide Volume. World Neurosurg. 2018;113:e345-e63. 45. Fischer PE, Perina DG, Delbridge TR, Fallat ME, Salomone JP, Dodd J, et al. Spinal Motion Restriction in the Trauma Patient - A Joint Position Statement. Prehospital emergency care : official journal of the National Association of EMS Physicians and the National Association of State EMS Directors. 2018:1-3. 